

\section{DISCLAIMER}

This report was prepared as an account of work sponsored by an agency of the United States Government. Neither the United States Government nor any agency Thereof, nor any of their employees, makes any warranty, express or implied, or assumes any legal liability or responsibility for the accuracy, completeness, or usefulness of any information, apparatus, product, or process disclosed, or represents that its use would not infringe privately owned rights. Reference herein to any specific commercial product, process, or service by trade name, trademark, manufacturer, or otherwise does not necessarily constitute or imply its endorsement, recommendation, or favoring by the United States Government or any agency thereof. The views and opinions of authors expressed herein do not necessarily state or reflect those of the United States Government or any agency thereof. 


\section{DISCLAIMER}

Portions of this document may be illegible in electronic image products. Images are produced from the best available original document. 


\title{
EQUATIONS OF STATE FOR THE DETONATION PRODUCTS OF HEXANITROSTILBENE AT VARIOUS CHARGE DENSITIES
}

\author{
by
}

E. L. Lee, J. R. Walton

Lawrence Livermore Laboratory

Livermore, California 94550

\author{
P. E. Kramer \\ Pantex Plant \\ Mason \& Hanger-Silas Mason Company \\ Amari110, Texas 79105
}

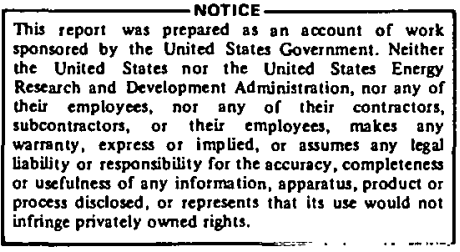




\section{ABSTRACT}

We have attempted here to provide an extensive description of the detonation behavior for the unique and useful high explosive hexanitrostilbene (HNS). To accomplish this we have compiled and evaluated the necessary experimental results measured by detonation of the pure material at charge densities of $1.00,1.20,1.40,1.60$, and $1.65\left(\mathrm{~g} / \mathrm{cm}^{3}=\mathrm{Mg} / \mathrm{m}^{3}\right)$. Estimates of the equation of state of the detonation products were made for each charge density. To confirm these estimates we carried out twodimensional hydrodynamic (HEMP code) calculations to simulate the cylinder test experiments for two charge densities of 1.2 and $1.6 \mathrm{Mg} / \mathrm{m}^{3}$. Detailed comparisons of the calculational and experimental results were made for these two tests.

Interpolation and extrapolation of the equation of state parameters provided final estimates for the other charge densities. The results are summarized in five sets of Chapman-Jouguet parameters and JWL equation of state coefficients.

\section{INTRODUCTION}

Because hexanitrostilbene (HNS) is chemically stable at elevated temperature and possesses relatively high shock sensitivity, it has become an important explosive material especially in initiator and booster applications. It is important in many of these applications to have an accurate estimate of its performance ( $i . e$, the duration and magnitude of the impulse delivered by the detonation of the charge). Considerable experimental work $1,2,3$ on HNS has been performed in order to provide this information.

We have evaluated these data and summarized the explosive performance in a set of equation of state coefficients. 4 This equation of state, used in hydrodynamic calculations, predicts the behavior of HNS at various charge densities for almost any charge geometry. The description presented applies directly to charge sizes sufficiently large to ignore edge effects on the detonation wave. 
The conditions for direct applicability are

$$
1-\frac{D}{D \infty}<\delta
$$

where $\delta=0.01$ is sufficiently small for most applications. ${ }^{*}$ In most applications the error introduced in ignoring edge effects is negligible. For precise predictions in situations where this criterion is not met provision must be made in the hydrodynamic model to account for the reaction zone behavior.

\section{EXPERIMENTAL RESULTS}

The experimental and calculational methods used to determine the equation of state have been described by Lee, et al, ${ }^{4}$ and much of the experimental data on HNS has been described previously. ${ }^{2,3}$ The discussion here will be primarily directed toward the estimation of the $\mathrm{C}-J$ pressures and detonation energies.

On the basis of $P_{C J}$ measurements on TNT we have reason to question Slape's ${ }^{2} P_{C J}$ results on HNS. The chemical energy and chemical composition of HNS and TNT are very similar. We have chosen to adjust the pressures for HNS upward to fall within the generally accepted range of values for. TNT.

For similar materials such as HNS and TNT a good estimate of the ratio of their $\mathrm{C}-\mathrm{J}$ pressures can be obtained from the quantity $K=\rho_{0} D^{2}$. The values obtained for TNT are $D=6.93 \mathrm{~mm} / \mu \mathrm{S}$ at $\rho_{0}=1.63 \mathrm{Mg} / \mathrm{m}^{3}$ and for HNS $D=7.03 \mathrm{~mm} / \mu \mathrm{s}$ at $\rho_{0}=1.65 \mathrm{Mg} / \mathrm{m}^{3}$ giving a ratio of 1.04 for $K(H N S) / K(T N T)$. The average Chapman-Jouguet pressure for TNT computed from a list (see Table 4) of reported values adjusted to a density of 1.63 is $207 \mathrm{kbar}(20.7 \mathrm{GPa})$. The resulting estimate of $\mathrm{C}-\mathrm{J}$ pressure for $\mathrm{HNS}$ at $\rho_{0}=1.65$ is $215 \mathrm{kbar} 21.5 \mathrm{GPa}$. The pressures estimated for densities of $1.6,1.4,1.2$, and $1.0 \mathrm{Mg} / \mathrm{m}^{3}$ were obtained from a $\mathrm{C}-\mathrm{J}$ pressure curve (determined by fitting the reported TNT $C-J$ pressure values and maintaining the same shape as the more precise $P_{C J}$ versus density dependence for $H M X)$. The results are shown in Figure 1.

For HNS the calculated heat of detonation $\Delta \mathrm{H}_{\mathrm{d}}$ based on. $\mathrm{H}_{2} \mathrm{O}$ as a gaseous detonation product is $5.69 \mathrm{MJ} / \mathrm{kg}$. The value calculated for $\Delta H_{d}($ TNT) calc

${ }^{\star}{ }^{\infty}$ is the detonation velocity for infinite charge size. 
is $5.40 \mathrm{MJ} / \mathrm{kg}$. Since an experimental value (detonation calorimetry) is not available for HNS we have adjusted the experimental value for TNT ${ }^{5}$ $\left(\Delta H_{d}(T N T)_{\text {exp }}=4.27\right)$ by the factor $\Delta H_{d}(\text { HNS })_{c a l c} / \Delta H_{d}(T N T)_{c a l c}$ to provide an estimate for the experimental value for HNS, giving $\Delta H_{d}(H N S)_{\text {exp }}=4.5 \mathrm{MJ} / \mathrm{kg}$. In addition we have made a correction corresponding to the difference in results found in calorimetry experiments between high density and low density high explosive charges. ${ }^{6}$ The heat of detonation at a density of $1.00 \mathrm{Mg} / \mathrm{m}^{3}$ is thereby reduced 10 percent. The value at a density of $1.65 \mathrm{Mg} / \mathrm{m}^{3}$ is as calculated above. The values at intermediate densities were interpolated.

\section{RESULTS}

The Chapman-Jouguet parameters and the JWL coefficients are listed in Table-1. The results of the experiments and the hydrodynamic calculation for the cylinder test are given in Table 2. Values for pressure volume and energy for isentropic expansion of the detonation products are listed in Table 3. Equation of state descriptions for intermediate densities can be obtained by interpolation between the values for the JWL coefficients $R_{1}$, $R_{2}$, and $W^{\star}$, and calculation of the necessary values for $A, B$, and $C$ to satisfy the appropriate Chapman-Jouguet conditions. ${ }^{4}$ We have been successfui in using the JWL equation of state in fitting the behavior of a large variety of explosives. The form of the JWL equation is:

$$
P=A\left(1-\frac{\omega}{R_{1} V}\right) e^{-R_{1} V}+B\left(1-\frac{\omega}{R_{2} V}\right) e^{-R_{2} V}+\frac{\omega E}{V},
$$

where $A, B, R_{1}, R_{2}$, and $\omega$ are constants, and $V$ is the relative volume $v / v_{0}$. The equation for $P$ as a function of $V$ at constant entropy is :

$$
P_{S}=A e^{-R_{j} V}+B e^{-R_{2} V}+C V^{-(\omega+1)}
$$

and the energy equation is:

$$
E_{S}=\frac{A e^{-R_{1} V}}{R_{1}}+\frac{B e^{-R_{2} V}}{R_{2}}+\frac{C}{{ }_{11} V^{\omega}} .
$$

${ }^{\star}{ }_{\omega}$ and $W$ are equivalent. 
Table 1. JWL Coefficients

\begin{tabular}{|c|c|c|c|c|c|c|}
\hline A & 1.627 & 2.631 & 3.665 & 4.606 & \multicolumn{2}{|l|}{4.631} \\
\hline B & 0.1082 & 0.07224 & 0.06750 & 0.07262 & \multicolumn{2}{|l|}{0.08873} \\
\hline$c$ & 0.006580 & 0.00945 & 0.01163 & 0.01338 & \multicolumn{2}{|l|}{0.01349} \\
\hline $\mathrm{R}_{1}$ & 5.4 & 5.0 & 4.8 & 4.60 & \multicolumn{2}{|l|}{4.55} \\
\hline $\mathrm{R}_{2}$ & 1.8 & $1: 5$ & 1.40 & 1.30 & \multicolumn{2}{|l|}{1.35} \\
\hline$W^{2}$ & 0.25 & 0.30 & 0.32 & 0.35 & \multicolumn{2}{|l|}{0.35} \\
\hline \multicolumn{7}{|c|}{ - C-J PARAMETERS } \\
\hline${ }^{*} \mathrm{P}_{\mathrm{CJ}}$ & 0.075 & 0.105 & .145 & 0.200 & 0.215 & Mbar \\
\hline Density & 1.00 & 1.20 & 1.40 & 1.60 & 1.65 & $\mathrm{Mg} / \mathrm{m}^{3}$ \\
\hline $\mathrm{D}$ & 5.10 & 5.74 & 6.34 & 6.95 & 7.03 & $\mathrm{~mm} / \mu \mathrm{s}$ \\
\hline * EO & 0.041 & 0.050 & .060 & 0.071 & 0.0745 & $\mathrm{Mbcc} / \mathrm{cc}$ \\
\hline$\Gamma$ & 2.468 & 2.765 & 2.881 & 2.864 & 2.804 & \\
\hline
\end{tabular}

*Systeme International Equivalence

$1.0 \mathrm{Mb}=100 \mathrm{GPa}$

$1.0 \mathrm{~Kb}=100 \mathrm{MPa}$

1.0 Mbcc $=10^{-4} \mathrm{GPa} \cdots \mathrm{m}^{3}=10^{-4} \mathrm{GJ}=100 \mathrm{MJ}$

1.0 $\mathrm{Mbcc} / \mathrm{cc}=100 \mathrm{GPa}: \mathrm{Mg} / \mathrm{m}^{3}=100 \mathrm{GPa}$ 
Table 2.1. Calculated and Experimental Cylinder Test Results HNS Density $=1.60 \mathrm{Mg} / \mathrm{m}^{3}$

\begin{tabular}{lccllll}
\hline R-RO cm & TEXP $\mu \mathrm{S}$ & TCALC & DT & VEXP $\mathrm{cm} / \mu \mathrm{S}$ VCALC & DV \\
\hline 0.00 & 0.00 & & & 0.0000 & & \\
0.05 & 0.79 & 1.0068 & -0.2168 & 0.0579 & 0.0588 & -0.0009 \\
0.10 & 1.65 & 1.7154 & -0.0554 & 0. & 0.0817 & 0. \\
0.15 & 2.30 & 2.3111 & -0.0111 & 0. & 0.0871 & 0. \\
0.20 & 2.80 & 2.8545 & -0.0545 & 0. & 0.0967 & 0. \\
\hline 0.25 & 3.31 & 3.3587 & -0.0487 & 0. & 0.1002 & 0. \\
0.30 & 3.88 & 3.8473 & 0.0327 & 0. & 0.1061 & 0. \\
0.35 & 4.39 & 4.3094 & 0.0806 & 0. & 0.1092 & 0. \\
0.40 & 4.76 & 4.7641 & -0.0041 & 0.1122 & 0.1115 & 0.0007 \\
0.50 & 5.64 & 5.6334 & 0.0066 & 0.1169 & 0.1165 & 0.0004 \\
\hline 0.60 & 6.47 & 6.4716 & -0.0016 & 0.1207 & 0.1213 & -0.0006 \\
0.70 & 7.30 & 7.2869 & 0.0131 & 0.1239 & 0.1250 & -0.0011 \\
0.80 & 8.10 & 8.0833 & 0.0167 & 0.1266 & 0.1271 & -0.0005 \\
0.90 & 8.88 & 8.3642 & 0.0158 & 0.1289 & 0.1288 & 0.0001 \\
1.00 & 9.65 & 9.6330 & 0.0170 & 0.1309 & 0.1306 & 0.0003 \\
\hline 1.10 & 10.41 & 10.3921 & 0.0179 & 0.1377 & 0.1324 & 0.0003 \\
1.20 & 11.16 & 11.1432 & 0.0168 & 0.1342 & 0.1340 & 0.0002 \\
1.30 & 11.90 & 11.8871 & 0.0129 & 0.1355 & 0.1352 & 0.0003 \\
1.40 & 12.63 & 12.6245 & 0.0055 & 0.1367 & 0.1362 & 0.0005 \\
1.50 & 13.36 & 13.3562 & 0.0038 & 0.1377 & 0.1371 & 0.0006 \\
\hline 1.60 & 14.08 & 14.0828 & -0.0028 & 0.1387 & 0.1380 & 0.0007 \\
1.70 & 14.81 & 14.8048 & 0.0052 & 0.1395 & 0.1388 & 0.0007 \\
1.80 & 15.51 & 15.5226 & -0.0126 & 0.1402 & 0.1396 & 0.0006 \\
1.90 & 16.23 & 16.2366 & -0.0066 & 0.1408 & 0.1404 & 0.0004 \\
2.00 & 16.94 & 16.9470 & -0.0070 & 0.1414 & 0.1411 & 0.0003 \\
\hline 2.20 & 18.35 & 18.3581 & -0.0081 & 0.1423 & 0.1423 & -0.0000 \\
2.40 & 19.76 & 19.7579 & 0.0021 & 0.1431 & 0.1434 & -0.0003 \\
2.60 & 0. & 21.1476 & 0. & 0. & 0.1444 & 0. \\
2.80 & 0. & 22.5285 & 0. & 0. & 0.1453 & 0. \\
3.00 & 0. & 23.9017 & 0. & 0. & 0.1460 & 0. \\
3.20 & 0. & 0. & 0. & 0. & 0. & 0. \\
\hline & & & & & & \\
\hline
\end{tabular}


$-6-$

Table 2.2 HNS Density $=1.2 \mathrm{Mg} / \mathrm{m}^{3}$.

\begin{tabular}{|c|c|c|c|c|c|c|}
\hline $\mathrm{R}-\mathrm{RO} \mathrm{cm}$ & TEXP $\mu \mathrm{S}$ & TCALC & DT & VEXP $\mathrm{cm} / \mu \mathrm{s}$ & VCALC & DV \\
\hline 0.00 & & 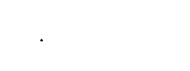 & & 0.0000 & & \\
\hline 0.05 & 1.31 & 1.2956 & 0.0144 & 0.0383 & 0.0468 & -0.0085 \\
\hline 0.10 & 2.26 & 2.2227 & 0.0373 & 0 & 0.0592 & 0 \\
\hline 0.15 & 3.02 & 3.0024 & 0.0176 & 0 & 0.0683 & 0 \\
\hline 0.20 & 3.72 & 3.7077 & 0.0123 & 0 & 0.0737 & 0 \\
\hline 0.25 & 4.37 & 4.3595 & 0.0105 & 0 & 0.0786 & 0 \\
\hline 0.30 & 4.98 & 4.9810 & -0.0010 & 0 & 0.0828 & 0 \\
\hline 0.35 & 5.56 & 5.5737 & -0.0137 & 0 & 0.0855 & 0 \\
\hline $0: 40$ & 6.15 & 6.1484 & 0.0016 & 0.0891 & 0.0887 & 0.0004 \\
\hline 0.50 & 7.24 & 7.2502 & -0.0102 & 0.0937 & 0.0932 & 0.0005 \\
\hline 0.60 & 8.29 & 8.3071 & -0.0171 & 0.0971 & 0.0965 & 0.0006 \\
\hline 0.70 & 9.31 & 9.3316 & -0.0216 & 0.0997 & 0.0991 & 0.0006 \\
\hline 0.80 & 10.30 & 10.3313 & -0.0313 & 0.1017 & 0.1012 & 0.0005 \\
\hline 0.90 & 11.27 & 11.3116 & -0.0416 & 0.1033 & 0.1030 & 0.0003 \\
\hline 1.00 & 12.24 & 12.2761 & -0.0361 & 0.1046 & 0.1045 & 0.0001 \\
\hline 1.10 & 13.19 & 13.2275 & -0.0375 & 0.1057 & 0.1058 & -0.0001 \\
\hline 1.20 & 14.13 & 14.1676 & -0.0376 & 0.1067 & 0.1070 & -0.0003 \\
\hline 1.30 & 15.06 & 15.0978 & -0.0378 & 0.1077 & 0.1080 & -0.0003 \\
\hline 1.40 & 15.99 & 16.0195 & -0.0295 & 0.1086 & 0.1090 & -0.0004 \\
\hline 1.50 & 16.90 & 16.9334 & -0.0334 & 0.1094 & 0.1099 & -0.0005 \\
\hline 1.60 & 17.81 & 17.8403 & -0.0303 & 0.1103 & 0.1107 & -0.0004 \\
\hline 1.70 & 18.72 & 18.7408 & -0.0208 & 0.1111 & 0.1114 & -0.0003 \\
\hline 1.80 & 19.61 & 19.6355 & -0.0255 & 0.1115 & 0.1121 & -0.0002 \\
\hline 1.90 & 20.51 & 20.5249 & -0.0149 & 0.1125 & 0.1128 & -0.0003 \\
\hline 2.00 & 21.39 & 21.4094 & -0.0194 & 0.1131 & 0.1134 & -0.0003 \\
\hline $2: 20$ & 23.15 & 23.1649 & -0.0149 & 0.1141 & 0.1145 & -0.0004 \\
\hline 2.40 & 24.90 & $\dot{0}$ & 0 & 0.1150 & 0 & 0 \\
\hline 2.60 & 0 & 0. & 0 & 0 & 0 & 0 \\
\hline 2.80 & 0 & 0. & 0 & 0 & 0 & 0 \\
\hline 3.00 & 0 & 0 & 0 & 0 & 0 & 0 \\
\hline 3.20 & 0 & 0 & 0 & 0 & 0 & 0 \\
\hline
\end{tabular}


Table 3:1 C-J Isentropic Pressures, Volumes and Energies HNS Charge Density $=1.65 \mathrm{Mg} / \mathrm{m}^{3}$

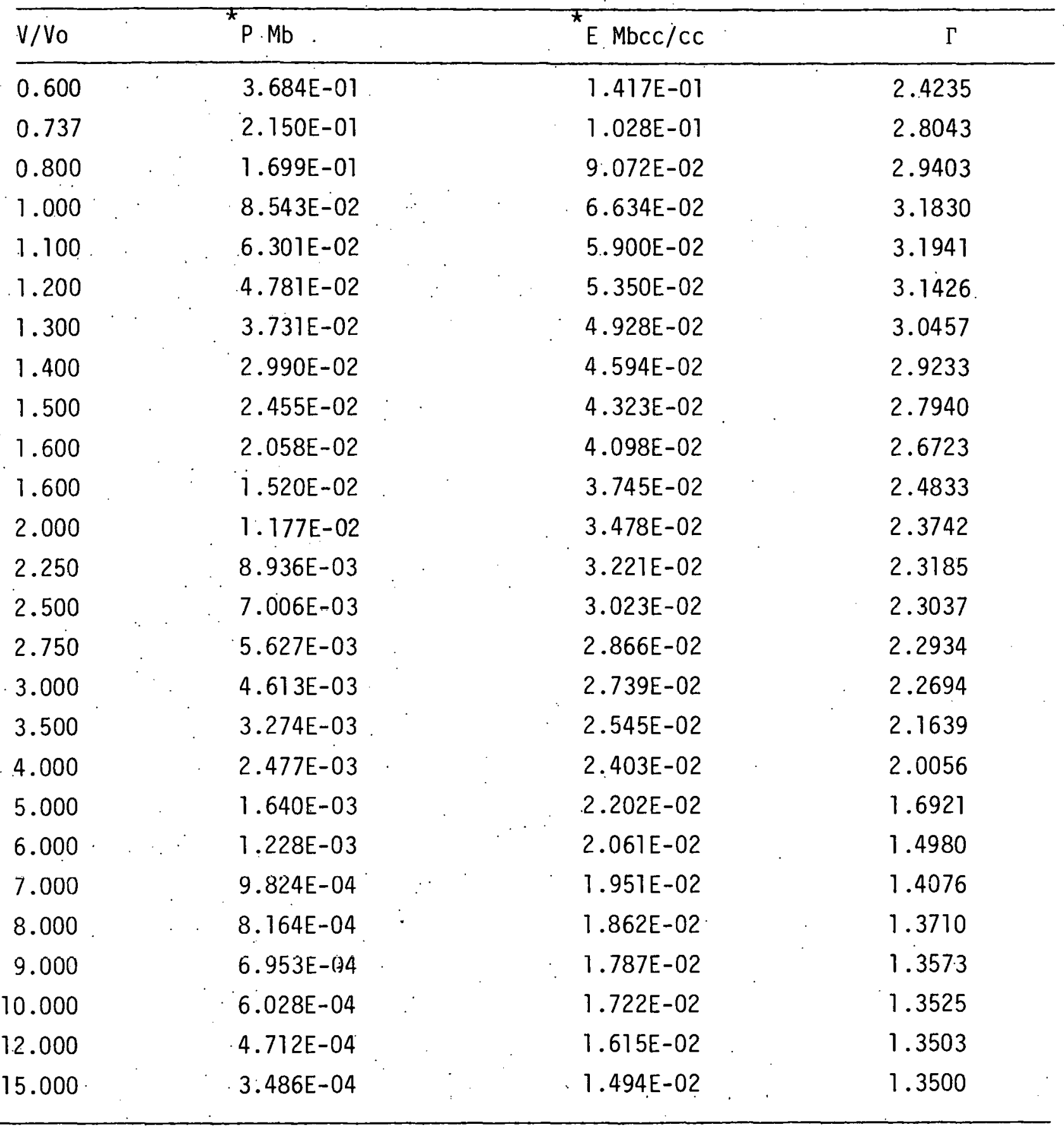

* See footnote, Tabīe 1 , page 4. 
Table 3.2

HNS Charge Density $=1.60 \mathrm{Mg} / \mathrm{m}^{3}$

\begin{tabular}{|c|c|c|c|}
\hline V/Vo & $\mathrm{P} M \mathrm{Mb}$ & $\mathrm{E} \mathrm{Mbcc} / \mathrm{Cc}$ & $\Gamma$ \\
\hline 0.600 & $3.575 \mathrm{E}-01$ & $1.347 \mathrm{E}-01$ & 2.4654 \\
\hline 0.741 . & $2.00 \mathrm{E}-01$ & $9.688 \mathrm{E}-02$ & 2.8642 \\
\hline 0.800 & $1.599 \mathrm{E}-01$ & $8.635 E-02$ & 2.9927 \\
\hline 1.000 & $7.947 \mathrm{E}-02$ & $6.353 \mathrm{E}-02$ & 3.23000 \\
\hline 1.100 & $5.837 \mathrm{E}-02$ & $5.671 \mathrm{E}-02$ & 3.2312 \\
\hline 1.200 & $4.418 \mathrm{E}-02$ & $5.163 \mathrm{E}-02$ & 3.1641 \\
\hline 1.300 & $3.444 \mathrm{E}-02$ & $4.773 \mathrm{E}-02$ & 3.0480 \\
\hline 1.400 & $2.762 E-02$ & $4.464 E-02$ & 2.9053 \\
\hline 1.500 & $2.272 \mathrm{E}-02$ & $4.214 \mathrm{E}-02$ & 2.7569 \\
\hline 1.600 & $1.910 \mathrm{E}-02$ & $4.006 \mathrm{E}-02$ & 2.6188 \\
\hline 1.800 & $1.422 E-02$ & $3.677 \mathrm{E}-02$ & 2.4063 \\
\hline 2.000 & $1.111 \mathrm{E}-02$ & $3.426 \mathrm{E}-02$ & 2.2857 \\
\hline 2.250 & $8.524 \mathrm{E}-03$ & $3.182 E-02$ & 2.2257 \\
\hline 2.500 & $6.748 E-83$ & $2.993 E-02$ & 2.2131 \\
\hline 2.750 & $5.465 E-03$ & $2.841 \mathrm{E}-02$ & 2.2088 \\
\hline 3.000 & $4.512 E-03$ & $2.717 \mathrm{E}-02$ & 2.1937 \\
\hline 3.500 & $3.235 \mathrm{E}-03$ & $2.5266-02$ & 2.1113 \\
\hline 4.000 & $2.461 \mathrm{E}-03$ & $2.385 \mathrm{E}-02$ & 1.9772 \\
\hline 5.000 & $1.633 \mathrm{E}-03$ & $2.186 \mathrm{E}-02$ & 1.6943 \\
\hline 6.000. & $1.221 \mathrm{E}-03$ & $2.045 \mathrm{E}-02$ & 1.5071 \\
\hline 7.000 & $9.758 \mathrm{E}-04$ & $1.936 \mathrm{E}-02$ & 1.4144 \\
\hline 8.000 & $8.103 E-04$ & $1.847 \mathrm{E}-02$ & 1.3747 \\
\hline 9.000 & $6.899 \mathrm{E}-04$ & $1.772 \mathrm{E}-02$ & 1.3590 \\
\hline 10.000 & $5 . .981 E-04$ & $1.700 E-02$ & 1.3532 \\
\hline 12.000 & $4.675 E-04$ & $1.603 \mathrm{E}-02$ & 1.3504 \\
\hline 15.000. & $3.459 \mathrm{E}-04$ & $1.482 \mathrm{E}-02$ & 1.3500 \\
\hline
\end{tabular}

* See footnote, Tahle 1 , page 4. 
Table 3.3

HNS Charge Density $=1.4 \mathrm{Mg} / \mathrm{m}^{3}$

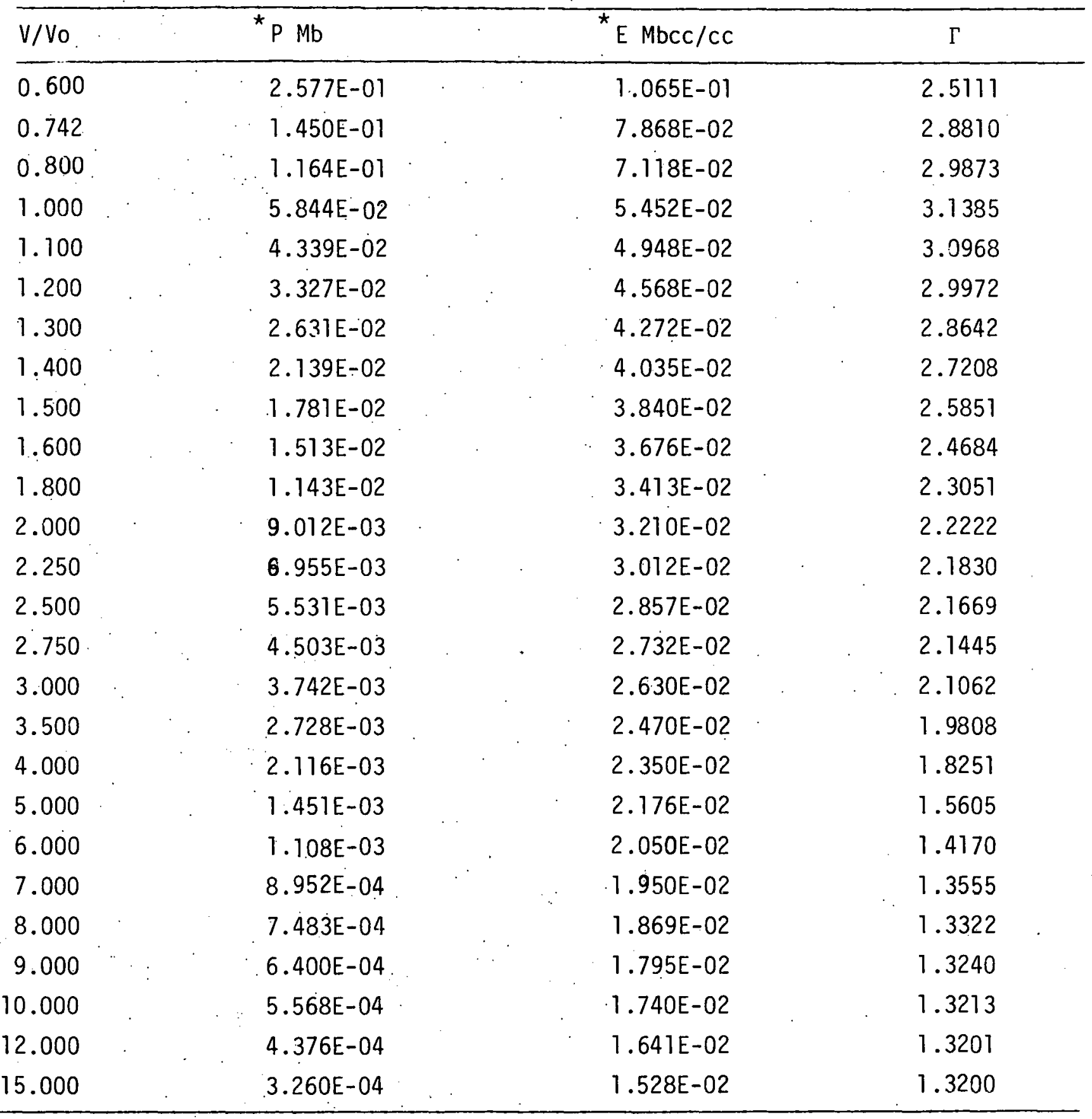

* See footnote, Table 1, page 4. 
Table 3.4

HNS Charge Density $=1.2 \mathrm{Mg} / \mathrm{m}^{3}$

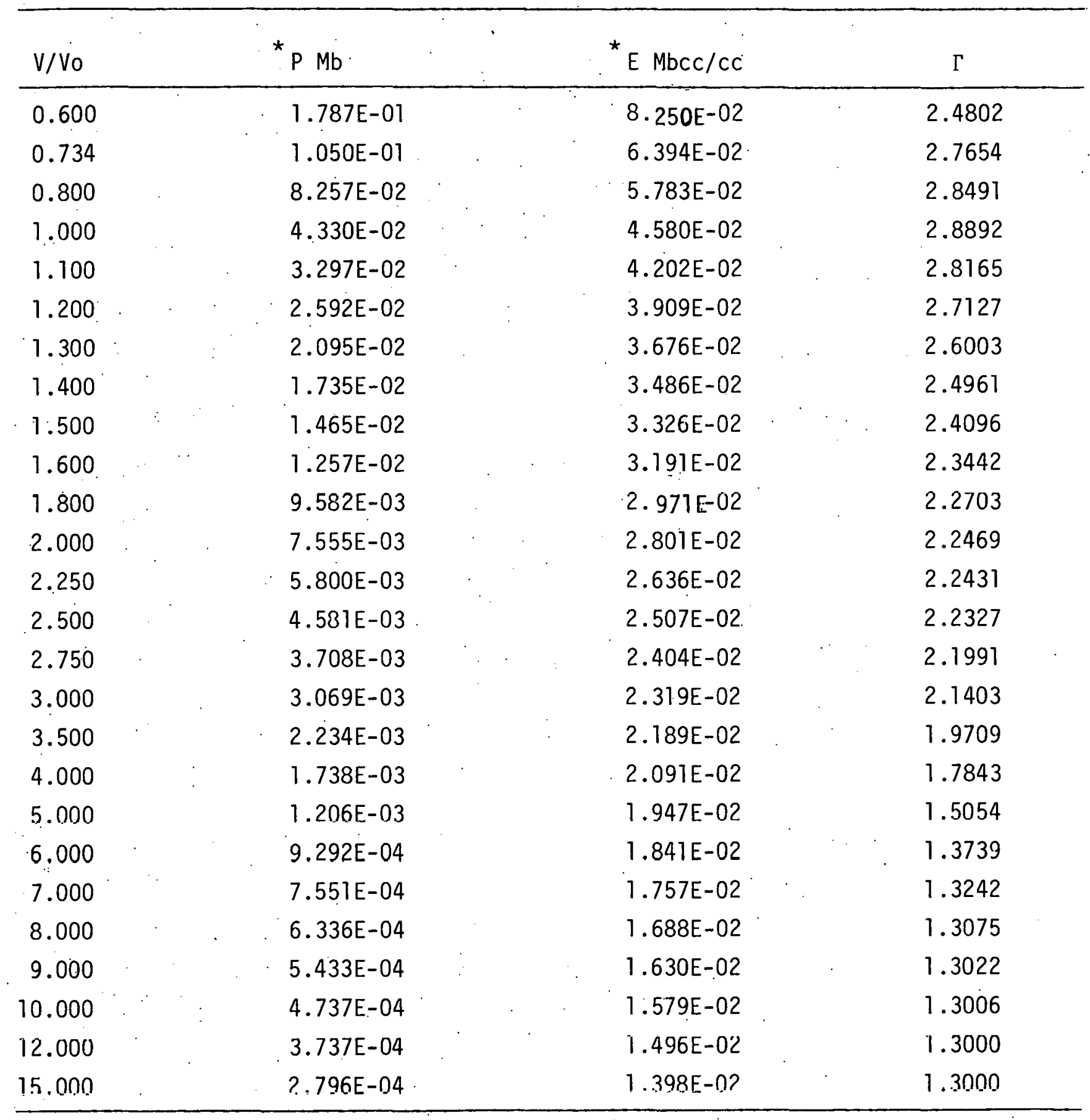

*Scc footnote, Table 1, page 4. 
Table 3.5

HNS Charge Density $=1.0 \mathrm{Mg} / \mathrm{m}^{3}$

\begin{tabular}{|c|c|c|c|}
\hline V/Vo & ${ }^{*} P M b$ & ${ }^{*} \mathrm{E} \mathrm{Mbcc} / \mathrm{cc}$ & $\Gamma$ \\
\hline 0.600 & $1.129 \mathrm{E}-01$ & $6.212 \mathrm{E}-02$ & 2.3175 \\
\hline 0.712 & $7.500 \mathrm{E}-02$ & $5.181 \mathrm{E}-02$ & 2.4680 \\
\hline 0.800 & $5.598 \mathrm{E}-02$ & $4.608 \mathrm{E}-02$ & 2.5239 \\
\hline 1.000 & $3.182 \mathrm{E}-02$ & $3.762 E-02$ & 2.5178 \\
\hline 1.100 & $2.507 \mathrm{E}-02$ & $3.479 \mathrm{E}-02$ & 2.4865 \\
\hline 1.200 & $2.022 E-02$ & $3.254 \mathrm{E}-02$ & 2.4575 \\
\hline $1.300^{\prime}$ & $1.662 E-02$ & $3.071 \mathrm{E}-02$ & 2.4387 \\
\hline 1.400 & $1.388 \mathrm{E}-02$ & $2.919 \mathrm{E}-02$ & 2.4324 \\
\hline 1.500 & $1.173 \mathrm{E}-02$ & $2.79 .1 \mathrm{E}-02$ & 2.4374 \\
\hline 1.600 & $1.002 \mathrm{E}-02$ & $2.683 \mathrm{E}-02$ & 2.4506 \\
\hline 1.800 & $7.492 \mathrm{E}-03$ & $2.509 \mathrm{E}-02$ & 2.4863 \\
\hline 2.000 & $5.757 \mathrm{E}-03$ & $2.378 \mathrm{E}-02$ & 2.5122 \\
\hline $2.250^{\circ}$ & $4.282 E-03$ & $2.254 \mathrm{E}-02$ & 2.5049 \\
\hline 2.500 & $3.298 E-03$ & $2.160 \mathrm{E}-02$ & 2.4433 \\
\hline 2.750 & $2.625 E-03$ & $2.086 \mathrm{E}-02$ & 2.3335 \\
\hline 3.000 & $2.155 \mathrm{E}-03$ & $2.027 \mathrm{E}-02$ & 2.1922 \\
\hline 3.500 & $1.573 \mathrm{E}-02$ & $1.935 \mathrm{E}-02$ & 1.8881 \\
\hline 4.000 & $1.244 \mathrm{E}-03$ & $1.865 E-02$ & 1.6365 \\
\hline 5.000 & $8.934 E-04$ & $1.761 \mathrm{E}-02$ & 1.3659 \\
\hline 6.000 & $7.029 E-04$ & $1.682 E-02$ & 1.2000 \\
\hline 7.000 & $5.782 E-04$ & $1.618 \mathrm{E}-02$ & 1.2572 \\
\hline 8.000 & $4.891 \mathrm{E}-04$ & $1.565 \mathrm{E}-02$ & $1 . .2516$ \\
\hline 9.000 & $4.221 \mathrm{E}-04$ & $1.519 E-02$ & 1.2504 \\
\hline 10.000 & $3.700 \mathrm{E}-04$ & $1.480 \mathrm{E}-02$ & 1.2501 \\
\hline 12.000 & $2.946 \mathrm{E}-04$ & $1.474 \mathrm{E}-02$ & 1.2500 \\
\hline 15.000 & $2.229 \mathrm{E}-04$ & $1.337 \mathrm{E}-02$ & 1.2500 \\
\hline
\end{tabular}

* See footnote, Table 1, page 4. 
Table 4a. C-J Pressure for TNT Density of $1.63 \mathrm{Mg} / \mathrm{m}^{3}$

\begin{tabular}{|c|c|c|c|c|c|c|}
\hline $\begin{array}{l}\text { Density }( \\
\mathrm{Mg} / \mathrm{m}^{3}\end{array}$ & & $\begin{array}{l}\text { Pressure } \\
k b^{*}\end{array}$ & $\begin{array}{l}\text { Pres } \\
\text { Adjuste }\end{array}$ & $\begin{array}{l}\text { sure } \\
\text { d to }\end{array}$ & $\begin{array}{l}k b * \\
\rho 1.63\end{array}$ & Reference \\
\hline 1.636 & & 188.4 & .. & 186 & & W. Deal J. Chem Phys 27,796 (1957) \\
\hline 1.614 & & 189 & $\cdot$ & 195 & & $\begin{array}{l}\text { Coleburn \& Liddiard J. Chem Phys } \\
1029, \underline{44}(1966)\end{array}$ \\
\hline 1.62 & . & 210 & $:$ & 213 & $\cdot$ & $\begin{array}{l}\text { Ilyukhin, Pakhil et a1. Daklad } 131 \\
793 \text { (1960) }\end{array}$ \\
\hline 1.622 & & 187.2 & & 190 & & Coleburn NOLTR 64-58 (1964) \\
\hline 1.62 & & 272 & & 215 & & $\begin{array}{l}\text { Y. Apin \& Voskoboynikov Pratk1. } \\
\text { Mekhan i Tekh. Fig } \underline{4} 54 \text { (1960) }\end{array}$ \\
\hline 1.632 & . & 213 & & & & R. Jameson BRL Private Communication \\
\hline 1.630 & & 220 & . & & & R. Crain Private Communication \\
\hline 1.63 & & 225 & & & & R. Craig 10th Comb. Symp. (1965) \\
\hline
\end{tabular}

Table 4b. Density Dependence

\begin{tabular}{lll}
$\begin{array}{l}\text { Density }(\rho) \\
\mathrm{Mg} / \mathrm{m}^{3}\end{array}$ & $\begin{array}{c}\text { Pressure } \\
\mathrm{kb}^{*}\end{array}$ & \multicolumn{1}{l}{ Reference } \\
\hline 1.44 & 178 & $\begin{array}{l}\text { W. Davis, B. Craig \& J. Ramsey } \\
4 \text { th Det. Symposium }\end{array}$ \\
1.08 & 115 & $\begin{array}{l}\text { Vevetennikov, Dremin, Shvedov } \\
\text { Fiz. Goreniya i Vsryva No. 3, } 3 \\
\text { (1965) }\end{array}$
\end{tabular}

\begin{tabular}{lll}
1.00 & 76.3 & \\
1.00 & 78.5 & 179 \\
1.59 & 64 & \multicolumn{1}{c}{$\begin{array}{l}\text { Dremin, Zaitzev, I1yukhin, } \\
\text { Pokhi1 8th Comb. Symp. 1960, } \\
610(1962)\end{array}$}
\end{tabular}

$\begin{array}{lr}1.14 & 94 \\ 1.30 & 123 \\ 1.45 & 162 \\ 1.59 & 202\end{array}$

* See footnote, Table 1, page 4. 


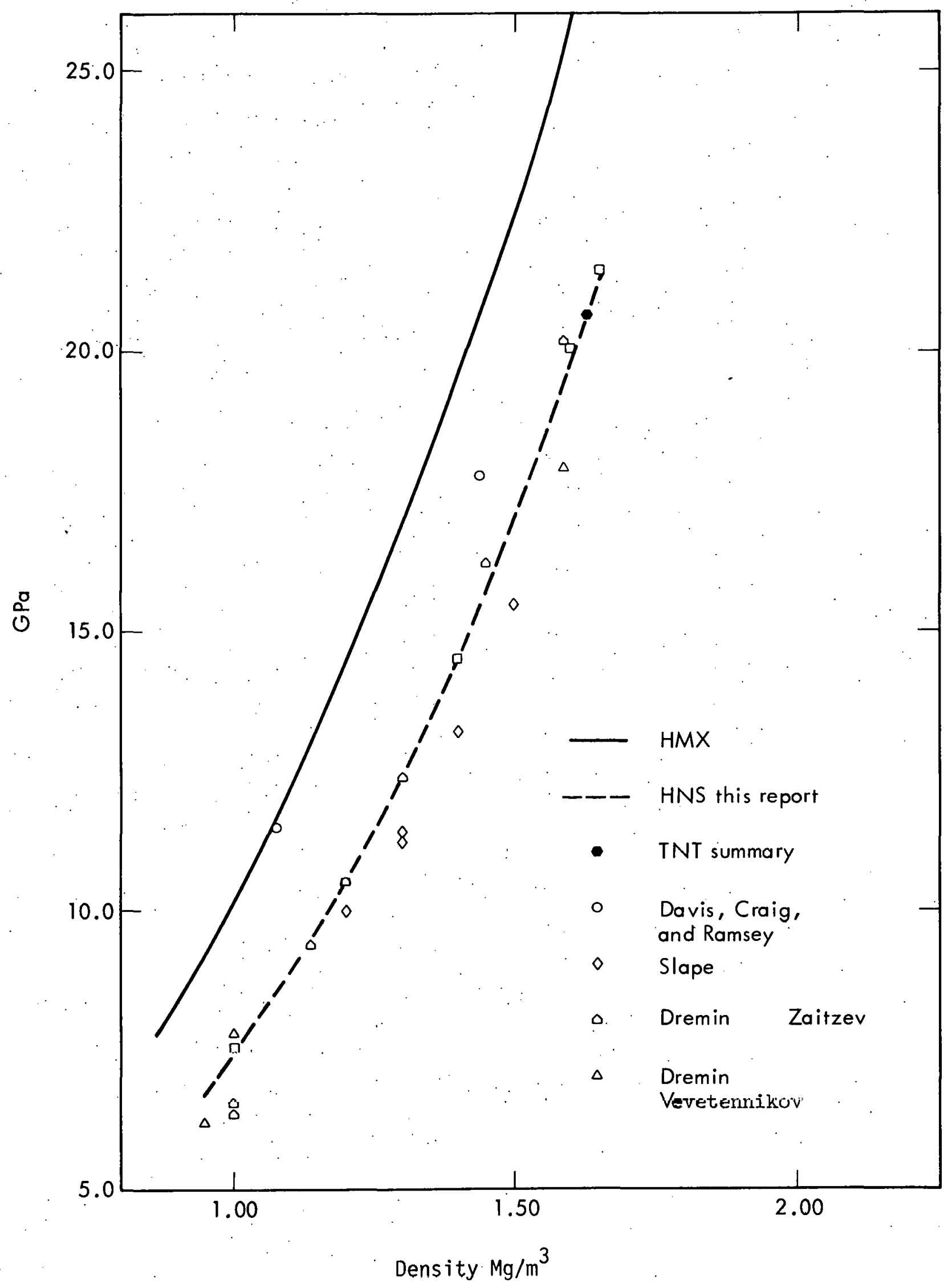

Figure 1. Detonation Pressures for HivS 


\section{REFERENCES}

1. L. D. Hanes, C. E: Canada, Unreacted hugoniots and Detonation Sensitivity of HNS I, MHSMP-75-25.

2. R. J. Slape, Detonation Pressure of HNS I and II, MHSMP-75-23.

3. P. E. Kramer, Development Division, HNS Cylinder Tests, MHSMP-75-17 (Apr 1975) Final Report (P. 0. No. 58-8474).

4. E. L. Lee, H. C. Hornig, J. W. Kury, Adiabatic Expansion of High Explosive Detonation Products, UCRL-50422 (May 2, 1968).

5. Brigitta M. Dobratz, Properties of Chemical Explosives and Explosive Simulants, UCRL-51319, MS. Date (December 15, 1972).

6. D. L. Ornellas, Lawrence Livermore Laboratory, The Heat and Products of Detonation in a Calorimeter of CNO, HNO, CHNF, CHNO, CHNOF, and CHNOSi Explosives, Combustion and Flame 23, 37-46 (1974). 


\section{LLL Internal Distribution}

H. Golopol

J. Stroud

D. Steinberg

M. Finger

R. McGuire

J. Walton

E. Lee (5)

D. Ornellas

L. Green

H. Hornig

B. Dobratz

TID (15)

TIC (27)

\section{External Distribution}

P. E. Kramer

R. S. Slape

Pantex Plant

Mason and Hanger

Silas Mason Co. Inc.

P. 0. Box 647

Amarillo, Texas 79177

D. Mitchel1 (5)

D. Hayes

Sandia Laboratories

P. 0. Box 5800

Albuquerque, New Mexico 87115

\section{Printed in the United States of America} Available from

National Technical Information Service

U.S. Department of Commerce 5285 Port Royal Road Springfield, Virginia 22151

Price: Printed Copy $\$$ *; Microfiche $\$ 2.25$

\begin{tabular}{c}
$*$ Pages \\
\hline $1-50$ \\
$51-150$ \\
$151-325$ \\
$326-500$ \\
$501-1000$
\end{tabular}

NTIS

Selling Price

$\$ 4.00$

$\$ 5.45$

$\$ 7.60$

$\$ 10.60$

$\$ 13.60$

\section{NOTICE}

"This report was prepared as an account of work sponsored by the United States Government. Neither the United States nor the United States Energy Research \& Develupuent Administration, nor any of their employees, nor any of their contractors, subcontractors, or their employees, makes any warranty, express or implied, or assumes any legal liability or responsibility for the accuracy, completeness or usefulness of any information, apparatus, product or process information, apparatus, product or process
disclosed, or represents that its use would not infringe privately-owned rights." 
Technical Information Department

LAWRENCE LIVERMORE LABORATORY

University of California | Livermcre, California |94£50 